\title{
Topological spaces and Abian structures
}

\section{Ralph Fox and Ivan L. Reilly}

This paper characterizes topological spaces which have an Abian structure - a uniform-like structure of the covering type.

In [1], Abian introduced a uniform-like structure of the covering type. More recently, Pu [6] has related such structures, which we call Abian structures following $\mathrm{Pu}$ 's lead, to a uniform-like structure of the entourage type, which he calls a regular structure.

DEFINITION 1. Let $X$ be a topological space and $\left\{A_{\lambda}: \lambda \in \Lambda\right\}$ a family of open covers for $X$. We define the $\lambda$-symmetric neighbourhood $S_{\lambda}(x)$ of $x$ by $S_{\lambda}(x)=U\left\{A \in A_{\lambda}: x \in A\right\}$. We also order $\Lambda$ by setting $\lambda \leq \mu$ iff $A_{\lambda}$ refines $A_{\mu} \cdot\left\{A_{\lambda}: \lambda \in \Lambda\right\}$ is called an Abian structure for $X$ if it satisfies:

(A.i) for every point $x$ and every open set $V$ containing $x$ there is some $\lambda \in \Lambda$ such that $U\left\{S_{\lambda}(y): y \in S_{\lambda}(x)\right\} \subseteq V$;

(A.ii) every two elements $\lambda$ and $\mu$ of $\Lambda$ are comparable; that is, given $A_{\lambda}$ and $A_{\mu}$, then either $A_{\lambda}$ refines $A_{\mu}$ or $A_{\mu}$ refines $A_{\lambda}$.

(Note that $\Lambda$ need not be totally ordered as $\leq$ need not be antisymmetric.)

Pu [6, Remark 4.2] showed that every topological space with an Abian structure must be regular, but was unable to settle the question as to

Received 8 March 1978. 
whether every regular topological space has an Abian structure.

In [2], we gave an example of a regular topological space which does not possess an Abian structure.

Perhaps a simpler example might be the Alexandroff one-point compactification $Y=X \cup\{\infty\}$ of an uncountable discrete space $X . Y$ is compact $T_{5}$, but the point $\infty$ does not have a totally ordered neighbourhood base and hence [2, Proposition] $Y$ does not possess an Abian stricture.

In this paper we give a necessary and sufficient condition for a topological space to possess an Abian structure. We show, in fact, a condition much like that for pseudometrizability, except that it does not imply first countability, namely the following:

CONDITION 1. The topological space $X$ is regular, and for some cardinal $k$ the following hold:

$$
\begin{aligned}
& \text { (B.i) } X \text { has a base } B=\bigcup_{k \in K} B_{k} \text { where } \# K=\kappa \text {, and for each } \\
& k \in K \text { every point of } X \text { has a neighbourhood meeting } \\
& \text { less than } \max \left\{k, k_{0}\right\} \text { members of } B_{k} ; \\
& \text { (B.ii) if } C \text { is a collection of open sets and } \# C<k \text { then } \\
& n\{C: C \in \mathcal{C}\} \text { is open. }
\end{aligned}
$$

Furthermore we show that $X$ has an Abian structure iff it has a compatible uniformity with a totally ordered base.

DEFINITION 2. We call a collection $\mathcal{C}$ of subsets of $X$ an $L_{\alpha}$ collection if every point of $X$ has a neighbourhood meeting less than $\mathbf{s}_{\alpha}$ members of $C$. Thus the $L_{0}$ collections are just the locally finite collections.

DEFINITION 3. We call a topological space $X$ with the property that the intersection of less than $\kappa_{\alpha}$ open sets is open, a $P_{\alpha}$ space.

Sikorski [7] has considered some of the properties of such spaces, and used the term $\omega_{\alpha}$-additive.

It should be observed that the notion of $P_{\alpha}$ space used by Misra [4] 
corresponds to our $P_{\alpha+1}$ space. It seems that Misra [4, footnote p. 349] is mistaken when he claims that his notion of $P_{\alpha}$ space coincides with Sikorski's $\omega_{\alpha}$-additivity. Thus Misra's $P$-spaces correspond to our $P_{1}$-spaces.

DEFINITION 4. We inductively define

$$
\begin{aligned}
S_{\lambda}^{l}(x) & =S_{\lambda}(x), \\
S_{\lambda}^{n+1}(x) & =\cup\left\{S_{\lambda}(y): y \in S_{\lambda}^{n}(x)\right\} .
\end{aligned}
$$

Note: $y \in S_{\lambda}^{n}(x)$ iff $x \in S_{\lambda}^{n}(y)$.

It may be easily shown that if $\left\{A_{\lambda}: \lambda \in \Lambda\right\}$ is an Abian structure for $X$, then if $U$ is an open set containing the point $x$, for any positive integer $n$ there is a $\lambda \in \Lambda$ such that $S_{\lambda}^{n}(x) \subseteq U$.

THEOREM 1. If $X$ has an Abian structure $\left\{A_{\lambda}: \lambda \in \Lambda\right\}$ then for some cardinal $k$ which is either $I$ or infinite regular ${ }^{1}, X$ satisfies Condition 1. Furthermore, $B=\bigcup_{k \in K} B_{k}$ may be chosen such that each $B_{k}$ is discrete.

Proof. We may suppose that $\Lambda$ is well-ordered by the reverse ordering $\geq$, and that ord $(\Lambda)$ is either $l$ or infinite regular. For if not, let $\Lambda^{\prime}$ be a coinitial subset of $\Lambda$ well-ordered by $\geq$, and suppose that ord $\left(\Lambda^{\prime}\right)$ has the minimum value possible. Then ord $\left(\Lambda^{\prime}\right)$ is either 1 or infinite regular, and $\left\{A_{\lambda}: \lambda \in \Lambda^{\prime}\right\}$ is an Abian structure for $X$.

Furthermore it is equivalent to $\left\{A_{\lambda}: \lambda \in \Lambda\right\}$ in that id $X$ is a uniform isomorphism between them; that is, is uniformly continuous [ $]$,

1 The regular cardinals are those cardinals $k$ which are not the sum of less than $k$ cardinals, each of which is less than $k$.

The regular ordinals are the initial ordinals whose corresponding cardinal is regular.

The infinite regular ordinals are characterized as being those infinite ordinals $\sigma$ with the property that if $S$ is a subset of $\sigma$ and $\operatorname{ord}(S)<\sigma$ then $S$ is not cofinal in $\sigma$. 
Definition 2] in both directions. Let $k=\# \Lambda$.

That topological spaces which have Abian structures, are regular was stated by $\mathrm{Pu}[6]$. Alternatively we may note that if $U$ is open and $x \in U$, then $S_{\lambda}^{2}(x) \equiv U$ for some $\lambda \in \Lambda$. Then

$$
x \in S_{\lambda}(x) \subseteq X-U\left\{S_{\lambda}(y): y \in X-U\right\} \subseteq U,
$$

$S_{\lambda}(x)$ is open, and $X-U\left\{S_{\lambda}(y): y \in X-U\right\}$ is closed, as required.

If $k=1$, then every point has a smallest neighbourhood. By regularity the topology is generated by a clopen partition $P$ of $X$. Clearly $P$ is the base required in (B.i). (B.ii) is trivially satisfied. Note that in fact in this case the arbitrary intersection of open sets is open. Spaces with this property have been considered by Pears [5] who calls them quasi-ordered spaces.

We now suppose $\kappa=\kappa_{\alpha}$ is infinite regular.

Let $K=\Lambda \times \Lambda$; then $\# K=\aleph_{\alpha}$. Let $\lambda \in \Lambda$, and irreflexively wellorder $A_{\lambda}$ by $<'$. For each $A \in A_{\lambda}$ and each $\mu \in \Lambda$ let

$$
\begin{aligned}
& A_{\mu}=\left\{x \in A: S_{\mu}^{4}(x) \subseteq A\right\}, \\
& A_{\mu}^{\prime}=A_{\mu}-\cup\left\{B \in A_{\lambda}: B<A^{\prime} A,\right. \\
& A_{\mu}^{*}=U\left\{S_{\mu}(x): x \in A_{\mu}^{\prime}\right\} .
\end{aligned}
$$

Then $\left\{A_{\mu}^{*}: A \in A_{\lambda}, \mu \in \Lambda\right\}$ is an open refinement of $A_{\lambda}$. We claim that for each $\mu \in \Lambda, B_{\lambda \mu}=\left\{A_{\mu}^{*}: A \in A_{\lambda}\right\}$ is discrete, and, in particular, that for each $x \in X, S_{\mu}(x)$ meets at most one $A_{\mu}^{*}$.

For suppose $S_{\mu}(x)$ meets $A_{\mu}^{*}$ and $B_{\mu}^{*}$. Then $S_{\mu}^{2}(x)$ meets $A_{\mu}$ and $B_{\mu}$. We may suppose $A<^{\prime} B$. Then let $y \in S_{\mu}^{2}(x) \cap A_{\mu}^{\prime}$, it follows that $S_{\mu}^{4}(x)$ meets $B_{\mu}^{\prime}$. But $y \in A_{\mu}$, so $S_{\mu}^{4}(x) \subseteq A$, and yet $B_{\mu}^{\prime} \cap A=\emptyset$. This is a contradiction, and hence $S_{\mu}(x)$ meets at most one $A_{\mu}^{*}$. 
We now claim $B=\underset{\lambda, \mu \in \Lambda}{U} B_{\lambda \mu}$ is a base. Suppose $x \in U$ and $U$ is open; then $S_{\lambda}(x) \subseteq U$ for some $\lambda$. Thus for some $\mu \in \Lambda, x \in A_{\mu}^{*} \in B_{\lambda \mu}$ where $A \in A_{\lambda}$, and so $x \in A_{\mu}^{*} \subseteq A \subseteq S_{\lambda}(x) \subseteq U$.

Thus $B=\underset{\lambda, \mu \in \Lambda}{U} B_{\lambda \mu}$ is the required base.

It remains to show that $X$ is a $P_{\alpha}$-space. Let $C$ be a family of open sets with $\# C<\aleph_{\alpha}$. If $n \mathcal{C} \neq \emptyset$, consider an arbitrary point $x \in \Pi C$. For each $c \in \mathcal{C}$ define $\lambda_{C}=\max \left[\lambda \in \Lambda: S_{\lambda}(x) \subseteq C\right]$. Then $\left\{\lambda_{C}: C \in C\right\}$ cannot be coinitial in $\Lambda$ as $\omega_{\alpha}$ is a regular ordinal, and hence there is some $\mu \in \Lambda$ such that $S_{\mu}(x) \subseteq C$ for all $C \in \mathcal{C}$. Hence nC is a neighbourhood of $x$.

Note 1. If $X$ satisfies Condition 1 then it does so for some cardinal $K^{\prime}$ which is either $I$ or infinite regular. For if $k$ is finite then $B$ itself is $L_{0}$ and so satisfies (B.i) with $K^{\prime}=1$. Hence Condition $l$ is satisfied with $K^{\prime}=1$.

We need now only consider $k$ infinite, but not regular.

Let $K=\aleph_{\alpha}$. Let $K^{\prime}$ be an index set with $K \subseteq K^{\prime}$ and $\# K^{\prime}=\aleph_{\alpha+1}$. Extend $\left\{B_{k}: k \in K\right\}$ to $\left\{B_{k}: k \in K^{\prime}\right\}$ by taking repetitions. Then $B=\bigcup_{k \in K^{\prime}} B_{k}$ is a base for $X$. Furthermore, the $B_{k}$ are $L_{\alpha} ;$ hence $L_{\alpha+1}$.

As $\aleph_{\alpha+1}$ is regular, it remains to show that $X$ is $P_{\alpha+1}$, for then Condition 1 will be satisfied with $\kappa^{\prime}=\kappa_{\alpha+1}$.

Let $\left\{U_{\gamma}: \gamma \in \Gamma\right\}$ be a family of less than ${ }^{*} \alpha+1$ open sets. We may assume $\# \Gamma=\kappa_{\alpha}$. Well-order $\Gamma$ so that $\operatorname{ord}(\Gamma)=\omega_{\alpha}$, and let $\Delta$ be a cofinal subset of $\Gamma$ of cardinal $\aleph_{\beta}<\aleph_{\alpha}$. For each $\delta \in \Delta$ let

$$
V_{\delta}=n\left\{U_{\gamma}: \gamma \in \Gamma, \gamma<\delta\right\} \text {. }
$$

Then each $V_{\delta}$ is open as it is the intersection of less than $\aleph_{\alpha}$ open 
sets. Furthermore,

$$
\bigcap_{\gamma \in \Gamma} U_{\gamma}=\bigcap_{\delta \in \Delta} V_{\delta}
$$

and $\prod_{\delta \in \Delta} V_{\delta}$, being the intersection of $\aleph_{\beta}\left(<\aleph_{\alpha}\right)$ open sets is open. Thus we see $X$ is also $P_{\alpha+1}$.

THEOREM 2. If $X$ satisfies Condition 1 then it is nomal.

Proof. If $k$ is finite, the topology is generated by a clopen partition of $X$, and so every closed set is also open. Hence $X$ is normal.

We now suppose $k=\aleph_{\alpha}$. Let $F$ and $G$ be disjoint closed sets, and well-order $k$ by $\leq$ so that $\operatorname{ord}(K)=\omega_{\alpha}$. Let

$$
\begin{aligned}
& U=\bigcup_{k \in K}^{U}\left(U\left\{B \in B_{k}: \bar{B} \cap G=\emptyset\right\}-\bigcup_{l \leq k}\left\{\bar{B}: B \in B_{l}, \bar{B} \cap F=\emptyset\right\}\right), \\
& V=\bigcup_{k \in K}\left(U\left\{B \in B_{k}: \bar{B} \cap F=\emptyset\right\}-\bigcup_{l \leq k}\left\{\bar{B}: B \in B_{l}, \bar{B} \cap G=\emptyset\right\}\right) .
\end{aligned}
$$

By regularity, $F \subseteq U, G \subseteq V$.

$X$ is a $P_{\alpha}$-space and $B_{k}$ is $L_{\alpha}$; hence $U\left\{\bar{B}: B \in B_{k}, \bar{B} \cap F=\emptyset\right\}$ is closed, and hence $\bigcup_{l \leq k} \cup\left\{\bar{B}: B \in B_{l}, \bar{B} \cap F=\emptyset\right\}$ is closed. Therefore $U$ is open. Similarly $V$ is open. Also, by construction, $U \cap V=\varnothing$. Hence $X$ is normal.

THEOREM 3. If $X$ satisfies Condition $I$ then it has a compatible uniformity with a totally ordered base.

Proof. We suppose $k$ is either 1 or infinite regular.

If $k \leq \kappa_{0}$ then $X$ is pseudometrizable, and the pseudometric uniformity has a (countable) totally ordered base.

If $k>\aleph_{0}$, say $k=\kappa_{\alpha}$ : for each $k \in K$ and $B \in B_{k}$ let $B_{Z}=U\left\{\bar{C}: C \in B_{Z}, \bar{C} \subseteq B\right\}$. By regularity and the fact that $B$ is a base we see that $\cup\left\{B_{Z}: Z \in K\right\}=B$.

$X$ is a $P_{\alpha}$ space and $B_{Z}$ is an $L_{\alpha}$ collection, so we may show that 
$B_{\mathcal{Z}}$ is closed. By Sikorski $[7, \S 2(\mathrm{v})]$ and the normality of $X$ we may find a clopen set $Q_{B Z}$ with $B_{Z} \subseteq Q_{B Z} \subseteq B$. Then $Q_{k Z}=\left\{Q_{B Z}: B \in B_{k}\right\}$ is $L_{\alpha}$ (but not necessarily a cover). Furthermore, $2=U\left\{Q_{k l}: k, l \in K\right\}$ is a base for the topology on $X$. Well-order $K \times K$ so that $\operatorname{ord}(K \times K)=\omega_{\alpha}$. Let $Q_{k}^{*} z^{\prime}=U\left\{Q_{k^{\prime}} Z^{\prime}:\left(k^{\prime}, Z^{\prime}\right) \leq(k, Z)\right\}$.

If $x \in X, x$ has a neighbourhood $W_{k} Z^{\prime}$ meeting no more. than $\aleph_{B}\left(k^{\prime}, l^{\prime}\right)$ members of $q_{k^{\prime}} z^{\prime}$, where $\aleph_{B}\left(k^{\prime}, z^{\prime}\right)<\aleph_{\alpha}$. Then as $\#\left\{\left(k^{\prime}, z^{\prime}\right):\left(k^{\prime}, Z^{\prime}\right) \leq\left(k, l^{\prime}\right)<\kappa_{\alpha}\right.$ and $X$ is a $P_{\alpha}$-space, $n\left\{W_{k^{\prime}} Z^{\prime}:\left(k^{\prime}, Z^{\prime}\right) \leq(k, l)\right\}$ is a neighbourhood of $x$ meeting no more than $\sum\left(\aleph_{\beta}\left(k^{\prime}, z^{\prime}\right):\left(k^{\prime}, z^{\prime}\right) \leq\left(k, z^{\prime}\right)\right)$ members of $2_{k^{\prime}}^{*}$. As $\aleph_{\alpha}$ is a regular cardinal, $\sum\left(\kappa_{\beta}\left(k^{\prime}, Z^{\prime}\right):\left(k^{\prime}, Z^{\prime}\right) \leq\left(k, l^{\prime}\right)<\kappa_{\alpha}\right.$. Thus $2_{k l}^{*}$ is an $L_{\alpha}$ collection.

Let $V_{k l}=\left\{(x, y): \forall Q \in Q_{k l}^{*}, x \in Q \Leftrightarrow y \in Q\right\}$. Then each $V_{k l}$ is transitive, and $v=\left\{V_{k l}:(k, \tau) \in K \times K\right\}$ is a totally ordered base for a uniformity on $X$. We claim that $V$ generates the topology on $X$.

Now $2=U\left\{q_{k}^{*} Z: k, Z \in K\right\}$, and is a base for the topology on $X$, and if $x \in Q \in Q_{k l}^{*}$ then $V_{k l}[x] \subseteq Q$. Hence it will suffice to show $V_{k Z}[x]$ is a neighbourhood of $x$.

As $2_{k Z}^{*}$ is $L_{\alpha}$, let $S$ be an open neighbourhood of $x$ meeting less than $\aleph_{\alpha}$ members of $2_{k l}^{*}$. Then $\left\{Q: Q \in Q_{k l}, x \in Q\right\}$ and $\left\{X-Q: Q \in Q_{k l}^{*}, Q \cap S \neq \emptyset, x \notin Q\right\}$ are two families of open neighbourhoods of $x$ of cardinal less than $\kappa_{\alpha}$. Let

$$
S^{*}=S \cap\left(\cap\left\{Q: Q \in 2_{k Z}^{*}, x \in Q\right\}\right) \cap\left(\cap\left\{x-Q: Q \in 2_{k}^{*}, Q \cap S \neq \varnothing, x \notin Q\right\}\right) .
$$

Then $S^{*}$ is an open neighbourhood of $x$. But $S^{*} \subseteq V_{k Z}[x]$ as required. Hence $V$ does generate the topology on $X$.

Note 2. If $V$ is a totally ordered base for a uniformity on $X$, 
then $\left\{A_{V}: V \in V\right\}$ is an Abian structure for $X$, where $A_{V}=\{$ int $V[x]: x \in X\}$.

This establishes the equivalence of possessing an Abian structure, satisfying Condition 1 , and possessing a compatible uniformity with a totally ordered base.

REMARK 1. By Theorem 3 and the result of Hayes [3], if $X$ satisfies Condition 1 , then it is paracompact. Furthermore if $k \neq \kappa_{0}$, then every open cover has a clopen partition refinement.

REMARK 2. If $X$ has Abian structure $\left\{A_{\lambda}: \lambda \in \Lambda\right\}$, where $\operatorname{ord}(\Lambda)$ is either 1 or infinite regular, then every point has a neighbourhood base well-ordered by $\supseteq$, for instance $\left\{S_{\lambda}(x): \lambda \in \Lambda\right\}$. For each point $x$ let on $(x)$ be the smallest ordinal of such a neighbourhood base. Then on $(x)$ is either 1 or ord $(\Lambda)$.

For clearly on $(x) \leq \operatorname{ord}(\Lambda)$ as the example $\left\{S_{\lambda}(x): \lambda \in \Lambda\right\}$ shows. But if $N$ is a neighbourhood base well-ordered by $\supseteq$, and $\operatorname{ard}(N)<\operatorname{ord}(\Lambda)$, then as $N$ is a $P_{\alpha}$-space, where $\operatorname{ord}(\Lambda)=\omega_{\alpha}, \cap N$ is a neighbourhood of $x$, and hence the smallest neighbourhood of $x$. Hence on $(x)=1$.

This gives a stronger analogue to first countability than might be expected.

REMARK 3. Connected topological spaces which possess Abian structures are pseudometrizable. For if $X$ is not pseudometrizable, then $\operatorname{ord}(\Lambda)>\omega_{0}$ and $X$ is not indiscrete. By regularity and Sikorski [7, $\S 2$ (iv)], $X$ is disconnected.

\section{References}

[1] Alexander Abian, "On uniform struetures", Rend. Circ. Mat. Palermo (2) 14 (1965), 202-206.

[2] Ralph Fox and Ivan L. Reilly, "Regular spaces and Abian structures", Rend. Circ. Mat. Palermo (2) 23 (1974), 267-270 (1976). 
[3] Al lan Hayes, "Uniformities with totally ordered bases have paracompact topologies", Proc. Cambridge Philos. Soc. 74 (1973), 67-68.

[4] Arvind K. Misra, "A topological view of P-spaces", General Topology and Appl. 2 (1972), 349-362.

[5] A.R. Pears, "On quasi-order spaces, normality, and paracompactness", Proc. London Math. Soc. (3) 23 (1971), 428-444.

[6] Hwang-Wen Pu, "Concerning a certain uniform structure", Rend. Circ. Mat. Palermo (2) 21 (1972), 98-102.

[7] Roman Sikorski, "Remarks on some topological spaces of high power", Fund. Math. 37 (1950), 125-136.

Department of Mathematics,

University of Auckland,

Auckland,

New Zealand. 